Journal of the

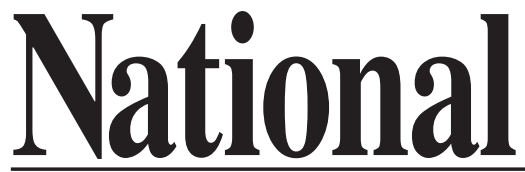

Academy or

Forensic
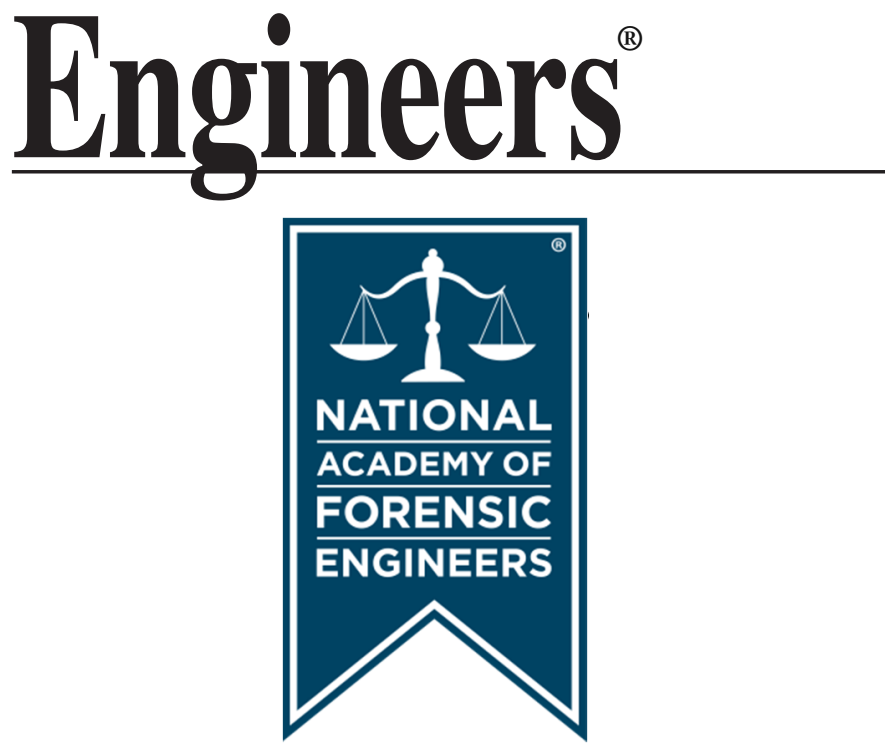

http://www.nafe.org

ISSN: 2379-3252

Vol. XXIII No. 2 December 2006 


\section{Forensic Engineering Use of Computer Animations and Graphics}

by Richard M. Ziernicki, Ph.D., P.E. (NAFE 308F)

David A. Danaher, P.E. (NAFE 703M)

\section{Introduction}

During an accident reconstruction investigation, many complicated issues arise that must be presented to a judge or jury. Although the Reconstructionist may understand the issues and can explain them orally, the audience may not completely understand. The use of computer animations and graphics can take those complicated issues and present them in a clear and understandable way. The graphics and animations that are developed from the engineering analysis are a visual representation of the Forensic Engineer's opinion. Psychological studies show that people are only $15 \%$ likely to remember orally-communicated information, but $95 \%$ likely to remember orally-communicated information in conjunction with visually-communicated information ${ }^{1}$. Visual tools, such as photo matching, evidence matching, photogrammetry, video matching, 3D modeling, simulation, and animation, can be implemented during the accident investigation process to identify evidence and demonstrate a thorough analysis for later use in court. For example, roof crush analysis graphics, as seen in Figure 1, not only visually illustrates the deformation of the roof's structure, but the graphics also have a solid foundation based on accurate measurements of the physical evidence.

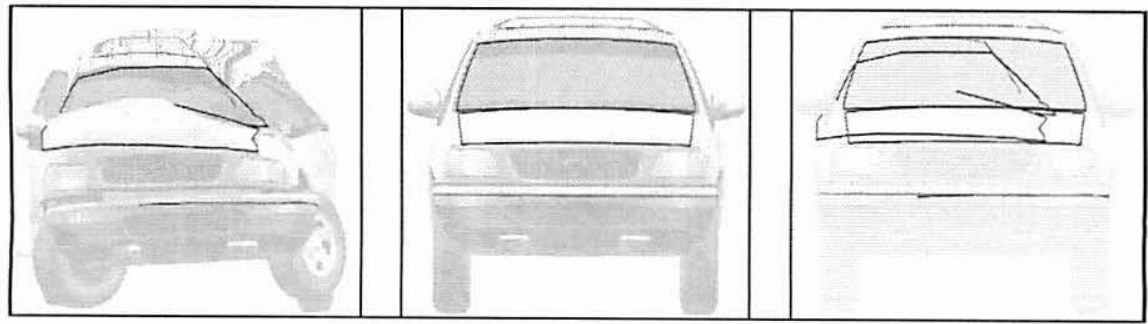

Figure 1

Roof crush analysis graphics depicts the amount of deformation between the exemplar vehicle and the accident vehicle. 
Copyright @ National Academy of Forensic Engineers (NAFE) http://www.nafe.org. Redistribution or resale is illegal.

\section{Documenting Physical Evidence at the Accident Site}

Prior to inspecting an accident site, the forensic engineer should review the available material, such as the police report, accident scene photographs, and witness statements. Along with reviewing the available material, the forensic engineer should also perform a preliminary analysis of the accident to determine the specific areas of interest involved in the case. This process of preliminary analysis prior to the inspection of the site or the vehicles can help the forensic engineer focus the investigation during the inspection. If all of the necessary physical evidence is documented during the inspection, the amount of time needed later in the reconstruction process can be greatly reduced.

When conducting a site inspection, the forensic engineer begins with the general documentation of the scene through photographs as well as locating physical evidence or rest positions documented by law enforcement officers. At times, the forensic engineer may be limited by the documentation provided by local law enforcement. Officials may not document specific details, such as gouges or marks left by the vehicle after impact. Other times some physical evidence may only be seen in the police photographs and may not be reported in detail in the police report. While at the site and equipped with the photographs, the engineer can also find additional physical evidence such as debris from the vehicle or evidence that may have been over looked. In addition to insufficient documentation, the site inspection may also take place several years after the accident occurred, leaving little to no physical evidence left at

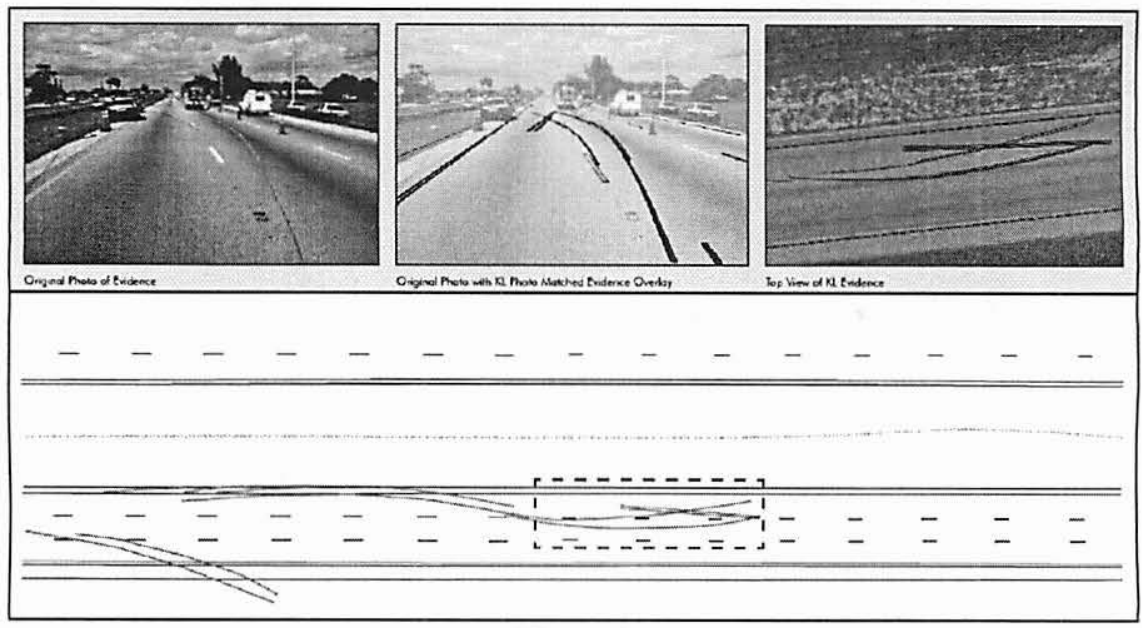

Figure 2

An accident scene photograph is matched up to the 3D surveyed terrain using a computer-generated camera to locate critical evidence from the accident, such as tire marks and gouges. 
the site. Although there is no physical evidence left, such as tire marks, documenting a roadway where the evidence was shown in police photographs can later generate a scene diagram.

After photographing the scene, a digital survey may be performed documenting evidence left on the road, along with physical attributes, such as painted lines, curbs, and seams. This detailed level of surveying can be used to generate a three dimensional scene diagram. The three-dimensional scene can then be matched, with a virtual camera, to the photographs taken by the police. See Figure 2. This visual, scientific method accurately locates specific details of the physical evidence such as the curvature and location of tire marks, the relative position of gouging in the roadway, the debris deposited on the ground as a result of impact, and the rest position of the vehicles.

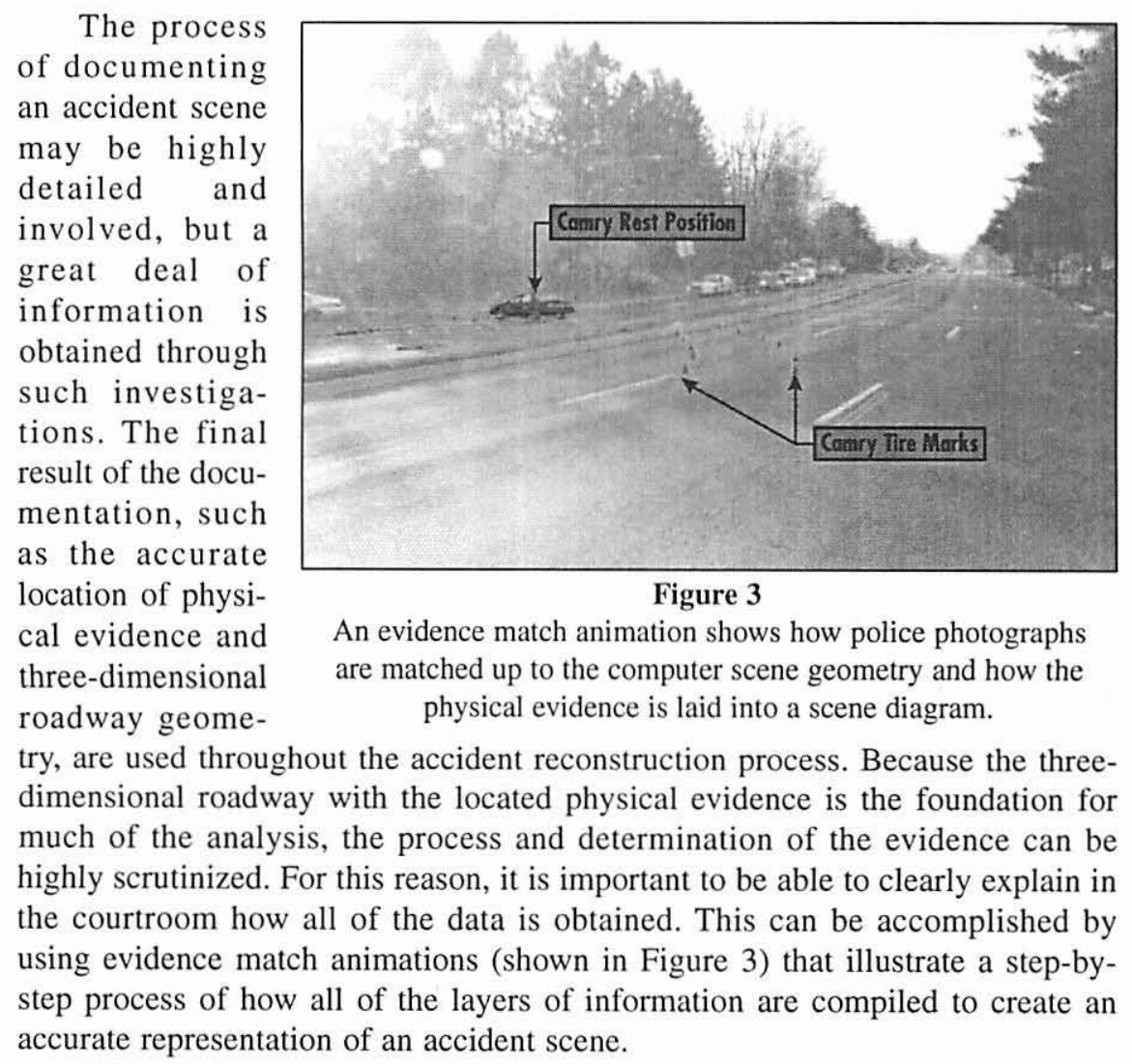




\section{Vehicle Damage Analysis}

In an accident reconstruction investigation, one of the first steps is to inspect the vehicles involved in the accident. The investigation team will document the vehicle's damage using photographs, measurements, and, when possible, a vehicle survey. After documenting the vehicle, specific photographs are taken that can be imported into computer software to determine the crush or deformation of the vehicle using the process of Photogrammetry. $[2,3,4,5]$ During the photogrammetry process, the user locates a minimum of three common points among a series of digital photographs of the damaged vehicle. When the photogrammetric process is complete the data can be compared with the vehicle survey data to determine the accuracy of the photogrammetry. In many investigations, the crush determined by Photogrammetry is compared to the crush obtained by the survey data collected during the vehicle inspection. A sample of this comparison is shown in Figure 4, showing that the difference between the photogrammetric data and the survey data was within several inches. These crush measurements are then analyzed by the engineer to determine impact speeds and impact severity using established methods of vehicle crush analysis.

Once the photogrammetric process is complete, the animation team will develop a scaled, 3D digital model of an exemplar vehicle, verifying the dimen-

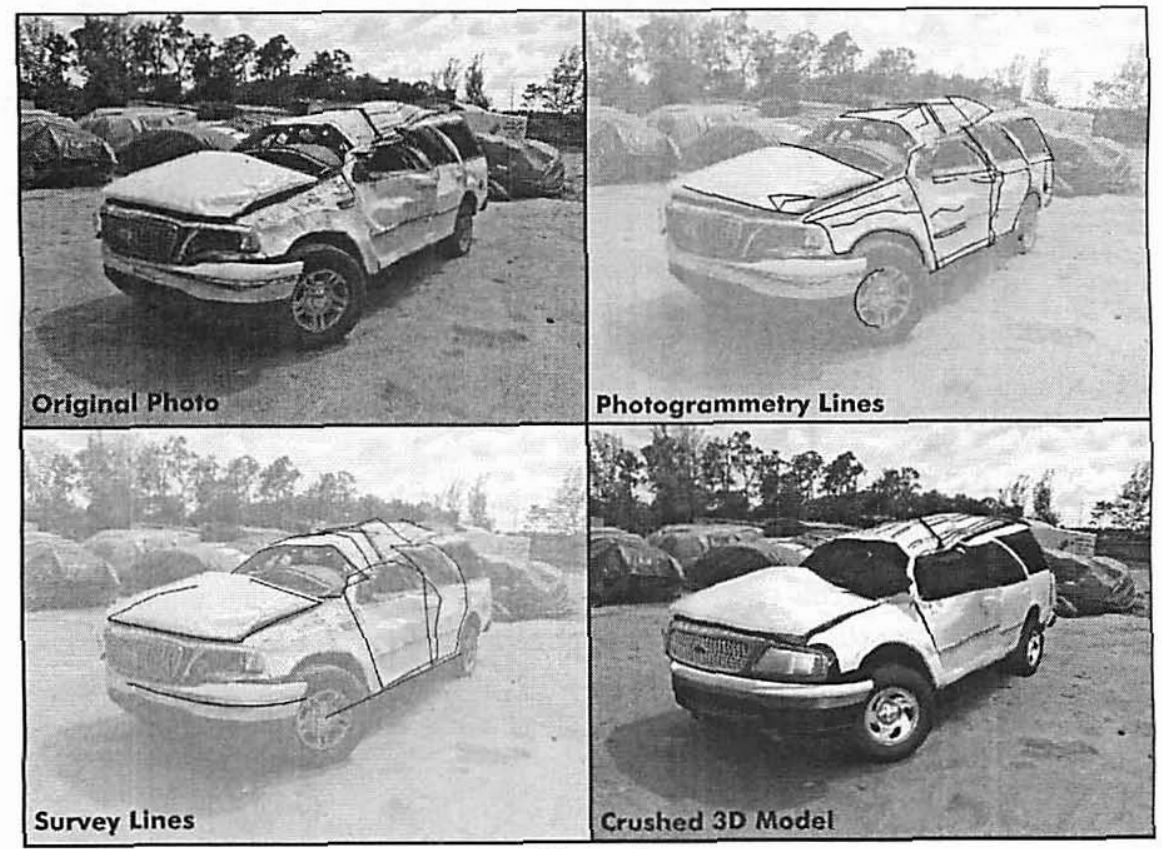

Figure 4

A comparison between the photogrammetry process and a vehicle survey 
sions with auto manufacturer specifications and photographs. The undamaged vehicle model is used in reconstruction animations and graphics, as well as to create the crushed model of the accident vehicle. The technique known as photo matching is used in conjunction with photogrammetry to make the crushed vehicle

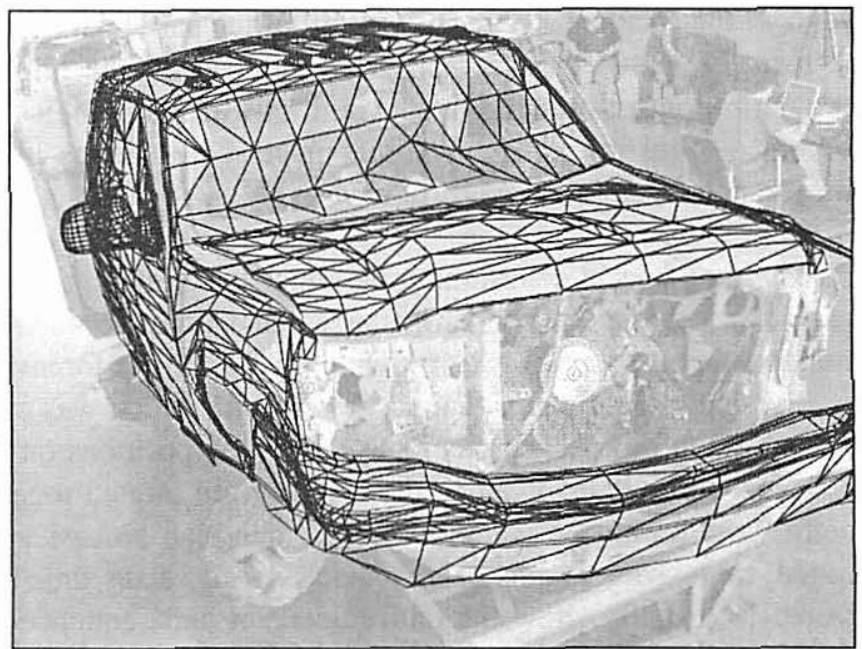

Figure 5

The photo matching technique is used to create a 3D computer mesh model of the damaged accident vehicle.

model. Photo matching is a process in which a virtual camera is positioned in the same location and with the same lens settings as the physical camera that took the photograph. Using the same series of photographs that were used in the photogrammetry process, the damaged vehicle photographs are matched in the computer (virtual space) using a 3D modeling program. Figure 5 shows an example of photo matching in which a computer mesh model, in wire frame mode, is "overlaid" on top of a photograph of the damaged vehicle. The 3D uncrushed vehicle model is now able to be crushed until the damage matches that of the accident vehicle, using photo matches and photogrammetry.

This modeling process is capable of compiling all of the complex vehicle damages, and of producing a 3D model that accurately shows vehicle crush profiles. These crushed models allow the forensic engineer to bring the damaged vehicle into the courtroom. Not only do the models represent an accurate representation of the vehicle damage, it also permits easy viewing by the judge and jury.

\section{Time-Space Analysis}

An important part of many forensic engineering accident analyses is the determination of time-space relationships between the vehicles involved in the accident. This analysis can involve complex relationships between vehicle speeds, acceleration or deceleration occurrences, driver perception and reaction times, and the location of vehicles at impact and at rest. When combined, these relationships will help the forensic engineer to determine the overall sequence of 
events of the accident. By combining all of the particular occurrences together in the form of scaled plots, graphs, and animations, the important issues of the accident become more readily apparent. The use of graphics and animations can be very helpful in expressing time-space relationships in both the reporting process as well as in the courtroom.

The first step in determining the time-space relationship is to use known or previously calculated speeds, distances, and times to determine the important intermediate positions of each individual vehicle. The forensic engineer can then use a scaled three-dimensional scene diagram, which was created earlier in the reconstruction process, to accurately plot these positions on the roadway. When all of the vehicles involved in the accident are plotted together relative to one another, the timing of events in the accident can be easier to visualize. When needed, time-space graphs of a vehicle's speed versus time and distance can be created to visually show the entire accident and sequence of events in a few illustrations. These types of scaled graphics and time-space graphs are particularly useful in expressing a large amount of information in a compact format.

When all of the relevant time-space information has been determined by the forensic engineer, it is often the case that the resulting analysis still remains rather complex. The subtleties of the accident may be difficult to express through still graphics. When this is the case, the forensic engineer can use a

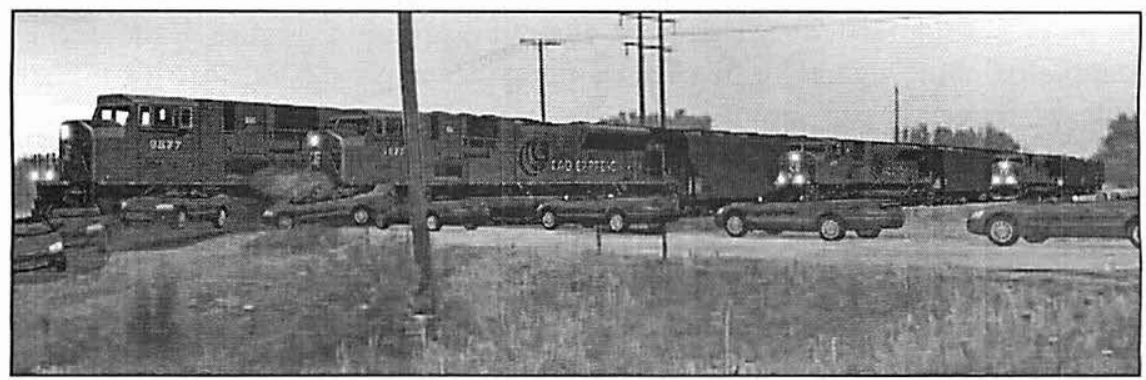

Figure 6

The overlaying frames from the animation show the complex interaction of vehicles involved in an accident.

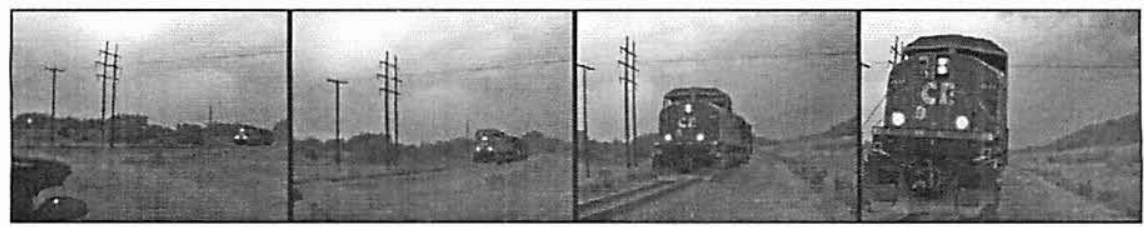

Figure 7

Frames taken from a video match animation show the driver's point of view leading up to the impact with the train. 
physics-based accident simulation software to show the motion of the vehicles. These simulations must be carefully compiled so that they are accurate portrayals of the vehicle motion in terms of speed, distance and time. This physicsbased software is then capable of producing the motion that forms the basis of an accurate 3D animation, as seen in Figure 6 and 7. One of the most useful aspects of the animation is that it represents the sequence of events in real time. The viewer is able to see exactly how the vehicles interacted over the same time period as when the accident actually occurred.

Animations can also be used to portray what a driver was capable of seeing during an accident. This is done by combining the computer simulation data with video shot from the driver's perspective, through a technique known as video matching. Figure 7 shows a series of frames taken from a video match of a driver's point of view, as his car approaches the railroad crossing. This is an effective way of portraying certain aspects of an accident, such as a driver's or witness' perception and reaction to the events.

The use of computer animations in the courtroom allows the judge and jurors to view a physically accurate portrayal of the complex dynamic interactions associated with a vehicle accident. Three-dimensional animations are an effective and easy to understand method of conveying the most important and relevant issues of an accident: such as the relative motion between the vehicles and their dynamic interaction.

\section{Examples of Graphic Applications in Litigation}

The three case studies below are examples of forensic engineering analysis and the application of graphics and animation and their effectiveness in litigation. The authors have extensive experience using computer animations and graphics in State and Federal court.

\section{Case 1:}

Semi vs. car

The accident occurred as the passenger vehicle made a left turn in front of a semi tractor-trailer at

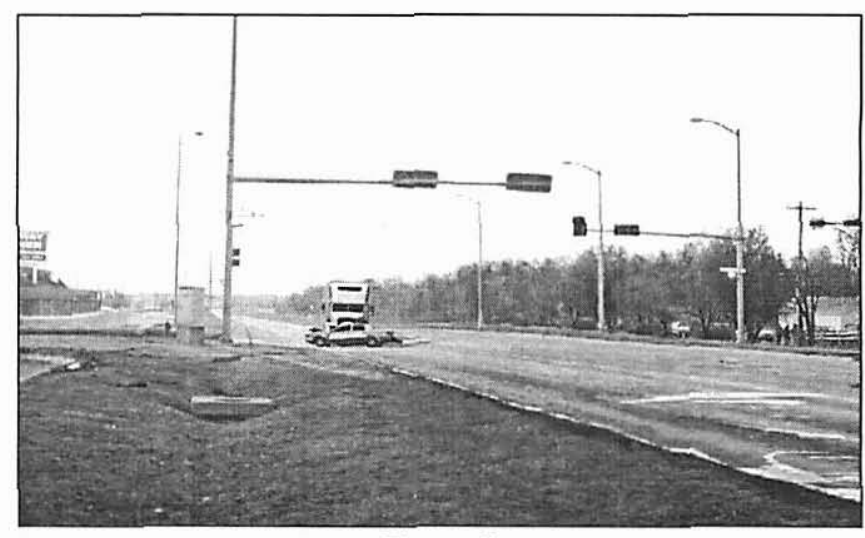

Figure 8

The photo matching technique is used to show the point of impact between the semi and the car. 
an intersection. An engineering investigation and analysis was performed to determine the position and speed of the vehicles. Once the analysis was complete, the information was used to develop animations that showed the semi driver's point of view as the vehicle traveled toward the intersection. Photo and video matching techniques were used in both the animations and graphics. The case went to Federal court and was Daubert challenged on both engineering analysis and animation issues. The Daubert challenge was unsuccessful and the engineer and the animations were allowed, by the Federal judge, to be presented to the jurors. Although the case was quite complex, the presentation to the jury was clear and concise.

\section{Case 2: Semi vs. Farm Equipment}

The accident occurred on a straight but hilly road in a rural area in Joplin, Missouri. A semi was traveling down the road and impacted the rear of a tractor towing a hay bailer. An engineering analysis and inspection was performed to determine any visibility issues for the semi driver. Analysis and inspection of the scene determined that there were no visibility obstructions for the semi driver. Once the analysis was complete, the information was used to develop several video matched anima-

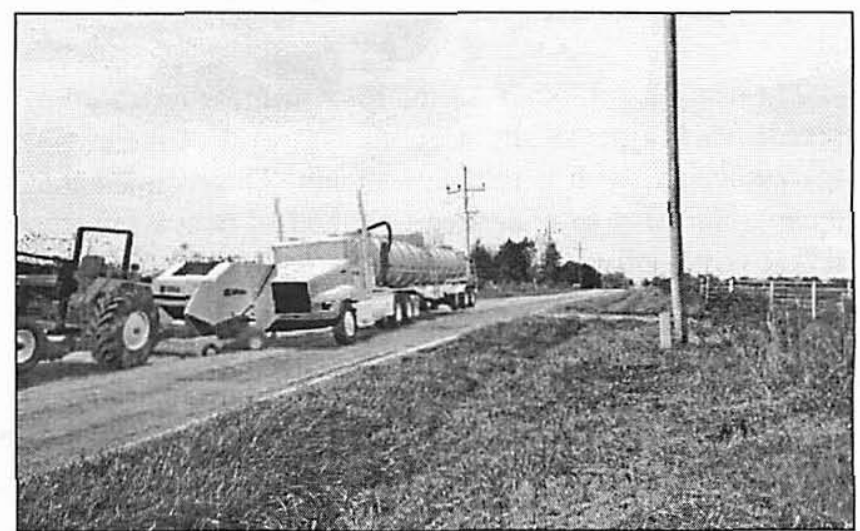

Figure 9

Animation clip showing a semi impacting the rear of a farm tractor towing a hay bailer.

tions which showed how long the farm tractor was visible to the semi driver. The case went to Federal court and all animations were Daubert challenged, but were admitted by the Federal judge. The engineer was able to testify and enhance his testimony with multiple animations and graphics.

\section{Case 3: Train vs. Car}

The accident happened during the day, in a rural area in Missouri. The passenger vehicle crossed a marked, but not gated, railroad crossing and the train hit the front of the car, propelling the vehicle off of the tracks. An engineering analysis and investigation was preformed at which time the accident scene and railroad tracks were surveyed and documented. Once the analysis was complete, video and photo matching techniques were used to reconstruct the train and car 
accident. The motion of the train through the accident scene was generated in virtual space and then "overlaid" with photos and videos taken at the accident site. The use of animation technology in generating the motion of the train colliding with the car was very effective and resulted in a settlement before the case went to trial. The clips from the animation are depicted in figure $6 \& 7$.

\section{Conclusion}

There are many aspects of a forensic engineering investigation that can be clearly and accurately conveyed in a visual manner. Visuals provide a powerful method of delivering important points to the viewer. Not only are the visuals "powerful" the advanced visual techniques, such as evidence matching and photogrammetry, can be used to locate evidence, which in turn is, used throughout the reconstruction process. These techniques are capable of producing graphics and animations that are scientifically based and highly detailed which provides credibility to the analysis.

Using the data collected through the discovery and investigation process, an engineer can analyze the dynamics of the crash and produce a physics-based simulation. This simulation provides a foundation for the production of computer animations of the accident. Computer animations and graphics can be effective in portraying all of the complex aspects of the case to a judge and jury in a simple and easy to understand format. Animations of an accident also allow all of the viewers to see one version of the accident, as opposed to each trying to visualize their own version based on the large amount of information provided. In this way, all 12 jurors are able to see the same accident depicted as they begin their deliberations.

\section{Acknowledgement}

The Authors would like to extend thanks to Hailey Day and Trevor Buss for their contribution in creating this paper. Their contributions include creation, enrichment and enhancement of this paper.

\section{References}

1. Pannish, B., Spagnoli, C., "Take Technology to Trial", Trial Magazine, July 2002

2. Ziernicki, Richard, and Stephen J. Fenton. "Two \& Three Dimensional Photogrammetric Accident Analysis." Journal of the National Academy of Forensic Engineers 17 No. 1 (Jun. 2000).

3. Fenton, Stephen J., Richard Ziernicki, Nathan Rose, and Wendy S. Johnson. "Using Digital Photogrammetry to Determine Crash Severity." International Journal of Crashworthiness REF: 2173 (2000).

4. Ziernicki, Richard, Stephen J. Fenton, Wendy S. Johnson, James 
LaRocque, and Nathan Rose. "Using Digital Photogrammetry to Determine Vehicle Crush and Equivalent Barrier Speed." Society of Automotive Engineers (SAE) paper 1999-01-0439 (1999).

5. Fenton, Stephen J., William Neale, Nathan Rose, and Christopher Hughes. "Determining Crash Data Using Camera Matching Photogrammetric Technique." Society of Automotive Engineers (SAE) paper 2001-01-3313 (2001).

6. Massa, David J. "Using Computer Reverse Projection Photogrammetry to Analyze an Animation." Society of Automotive Engineers (SAE) paper 1999-01-0093 (1999).

\section{Glossary of Terms}

Photo Matching - The process of matching the position and lens of a camera into 3D computer space from real world coordinates. Also known as camera matching.

Evidence Matching - Photo matching for the particular purpose of gathering evidence from photographs. Also known as Reverse Camera Projection (RCP) or scene photogrammetry.

Photogrammetry - A measurement technology in which the 3D coordinates of points on an object are determined by measurements made in two or more photographic images taken from different positions. Common points are identified on each image and a ray can be constructed from the camera location to the point of the object. The triangulation (intersection of the rays) determine the $3 \mathrm{D}$ location of the point.

Video Matching - The process of tracking the position of a moving camera in 3D computer space from real world coordinates.

3D Modeling - Building a digital version of a three-dimensional object to scale.

Simulation - A mathematical exercise in which a model of a system is established, then the model's variables are altered to determine the effects on other variables.

Animation - A motion picture that is made from a series of computer graphics that simulates movement by slight progressive changes in each frame. 